\title{
Sparse Channel Estimation using Hybrid Approach for OFDM Transceiver
}

\author{
Nisha Sachan \\ Department of Electronics \\ Engineering \\ Truba College of Science and \\ Technology, Bhopal, INDIA
}

\author{
Paresh Rawat \\ Department of Electronics \\ Engineering \\ Truba College of Science and \\ Technology, Bhopal, INDIA
}

\author{
Nashrah Fatima \\ Department of Electronics \\ Engineering \\ Truba College of Science and \\ Technology, Bhopal, INDIA
}

\begin{abstract}
Orthogonal Frequency Division Multiplexing (OFDM) is very sensitive to frequency offsets that is use to demolish the orthogonality among number of subcarriers. The OFDM introduced the inter-carrier interference and it is also capable of degrading the error performance. The channel estimation plays an important role to make OFDM more efficient. Proposed approach uses hybrid technique for Sparse Channel Offset (SCO) estimation in orthogonal frequency division multiplexing (OFDM) over frequency selective fading channel by using pilot tone and Hamming window filtering approach. Proposed approach uses hamming windows function in order to optimize Fast Fourier Transform channel estimation algorithms. Common window functions are: Rectangular window, Hanning window and Hamming window. Hamming window function as the main lobe has a good width and side-lobe decay rate. The result of the proposed approach is better than the previous approaches.
\end{abstract}

\section{Keywords}

OFDM, Channel Estimation, Pilot Carrier

\section{INTRODUCTION}

OFDM is being widely applied in wireless communications systems due to its high rate transmission capability with high bandwidth effectiveness and its sturdiness with regard to multi-path fading and delay. $\mathrm{WiFi}$ or 802.11 and HIPRLAN/2 uses the OFDM.OFDM has also been available for wireless broadband access standards such as IEEE standard WiMAX and as the core modus operandi for the fourth-generation $(4 \mathrm{G})$ wireless mobile communications. By using differential phase-shift keying (DPSK) in OFDM systems need to track a time varying channel is circumvented; however, it confines the number of bits per symbol and grades in a $3 \mathrm{~dB}$ loss in signal-to-noise ratio (SNR). Coherent modulation permits arbitrary signal constellations, but coherent detection and decoding call for efficient channel estimation strategies.

There are two main snags in designing channel estimators for wireless OFDM systems. The foremost delinquent is the prearrangement of pilot information, where pilot refers to the reference signal which is used by both transmitters and receivers. Next difficulty is the design of an estimator with both low complexity and good channel tracking capability. The two problems are interrelated. In general, the fading channel of OFDM systems can be watched as a two dimensional signal like time and frequency. The optimal channel estimator in terms of mean-square error is grounded on 2D Wiener filter interpolation. Tactlessly, such a 2D estimator structure is too multifaceted for real-world application. The amalgamation of high data rates and low bit error rates in OFDM systems compels the usage of estimators that have both low complexity and high accuracy, where the two constraints toil against each other and a decent trade-off is desirable. The one-dimensional channel estimations are usually accepted in OFDM systems to undertake the trade-off between complexity and accuracy. The two basic 1D channel estimations are block-type pilot channel estimation and comb-type pilot channel estimation, in which the pilots are implanted in the frequency direction and in the time direction, correspondingly. The valuations for the block-type pilot arrangement can be centered on least square (LS), minimum mean-square error (MMSE), and reformed MMSE. The estimations for the comb-type pilot arrangement contains the LS estimator with 1D interpolation, the maximum likelihood (ML) estimator, and the parametric channel modeling-based (PCMB) estimator. Additional channel estimation strategies are also available, such as the estimators built on shortened 2D interpolations, the estimators grounded on iterative riddling and decoding, estimators for the OFDM systems with multiple transmitand-receive antennas, and so on.In the presented paper Propose approach use DFT based hamming windows function in order to optimize FFT channel estimation algorithms, where common window functions are: rectangular window, Hanning window, and Hamming window. Hamming window function as the main lobe has a good width and side-lobe decay rate.

\section{ORTHOGONAL FREQUENCY DIVISION MULTIPLEXING}

The fast growth of data transfer at a high rate marks the need to advance network performance. This can be done easily by making use of Orthogonal Frequency Division Multiplexing. OFDM is the approach that has been the use of transfer of the technology of wireless multicarrier communications. Here was a high use of data for a data transfer rate. These may be high division data in an order different from data. These channels can be transmitted simultaneously by the Sub airlines. It includes the IEEE 802.11a OFDM standards as a criterion for sustainability in the fading light of the multiplicity of the conditions.Figure 1 illustrates the basic approach to save the channel by using OFDM. As in figure there are 7 different frequencies which can be send to the end user with some different approach known as OFDM. As shown in figure 1 the same channel can be used for some more different frequencies. 


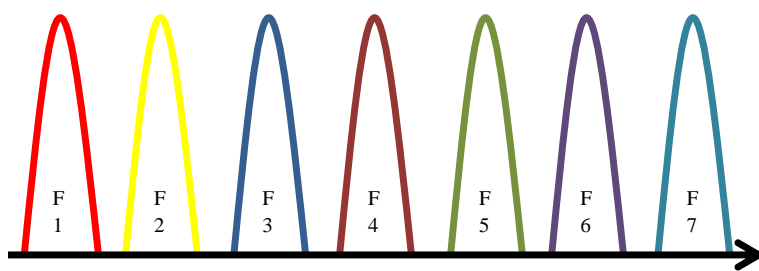

Frequency

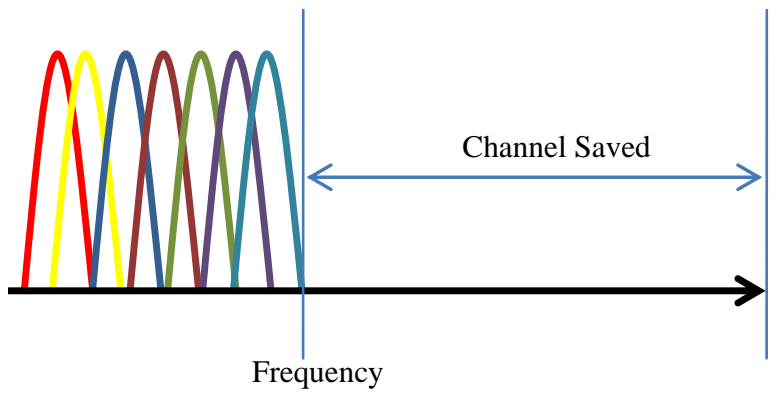

Figure 1: OFDM in Used

Figure 2 is explaining the basic block diagram of OFDM transmitter and receiver. There are five basic steps which are needed to follow in OFDM transmission and receive. First, the input binary sequence is inserted pilot symbols then modulation mapping is performed. The result of which goes to the IDFT modulator. Then cyclic prefix insertion is done and the signal is passed to parallel to serial converter. Similarly it follows the steps at receiver side.

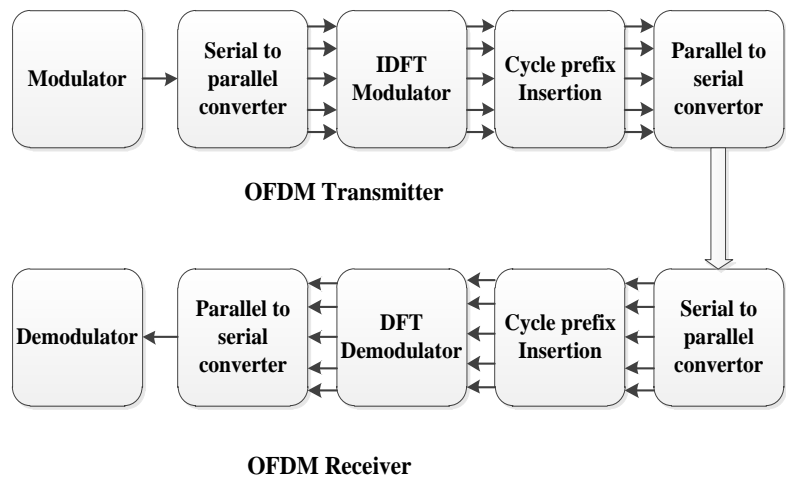

Figure 2: OFDM transmitter and Receiver

Wireless and mobile communication's applications of Orthogonal Frequency Division Multiplexing (OFDM) are presently under study. Although there are several substantial downsides of multi-carrier transmission, its recompenses in declining unadorned effects of frequency selective fading without a complicated equation is very smart feature. In order to gain high spectral efficiencies required by wireless data systems in the future, it is necessary to make use of multiple level modulation with changing amplitude. This means that there is prerequisite of coherent receiver which is able to track changes in the channel fading.

\section{CHANNEL ESTIMATION METHODS}

Channel estimation methods based on the pilot insertion can be classified into two classical pilot mockups, which are block-type and comb-type mockups. The first model states that the pilots are interleaved into all the subcarriers of one
OFDM symbol with a certain period. The block-type can be accepted for slowly fading channel, that is, the channel can be considered as immobile within a certain period of OFDM symbols. Nevertheless, the second model states that the pilots are positioned at some definite subcarriers in each OFDM symbol. Figure 3 (a,b) illustrates the two basic 1D channel estimations in OFDM systems. The block-type pilot channel estimation is technologically advanced under the postulation of slow fading channel, and it is executed by interleaving pilot tones into all subcarriers of OFDM symbols within a specific period. The other one, comb-type pilot channel estimation, is introduced to gratify the prerequisite for equalizing when the channel deviates even from one OFDM block to the consequent one. It is thus implemented by interleaving pilot tones into certain subcarriers of each OFDM symbol, where the interpolation is desirable to estimate the environments of data subcarriers.

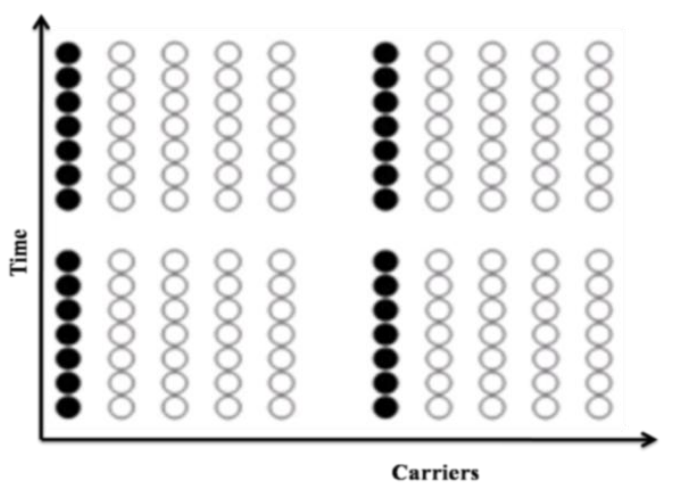

Figure 3(a): Comb Type

Above figure 3(a) shows the Comb Type channel estimation. In such attitude the part of the sub-carriers are always earmarked as pilot for each symbol.

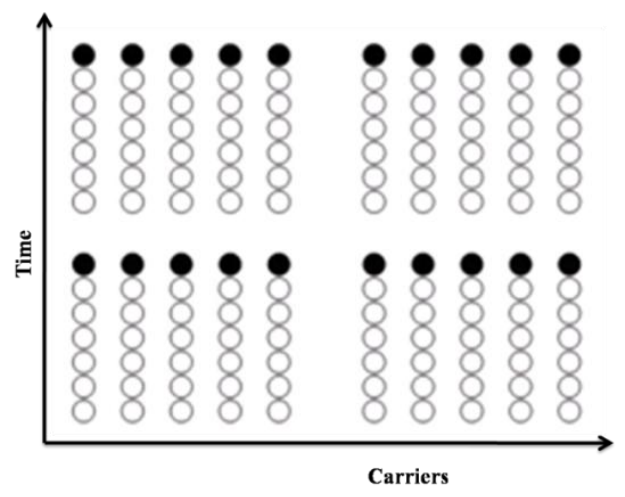

Figure 3(b): Block Type

The figure 3(b) shown above is Block Type channel estimation. Here all sub-carriers are used as pilot in a precise period.

\section{RELATED WORK}

There are various techniques which are developed to increase the efficiency of channel estimation under multipath environment. This section reviews the existing relevant research work.

Chenhao Qi[1] has proposed a scheme using the improved discrete stochastic approximation to optimize the pilot placement in OFDM systems. The channel data is employed to offline search the near-optimal pilot placement beforehand 
the transmission. In the interim author has also got a criterion to choose CS algorithms grounded on the mean squared error (MSE) minimization.Xiaochao[2] proposed channel estimation which can attain the channel fading information which is a key technology to expand the performance at the receive nodule in wireless channel transmission. The author has applied the compressed sensing theory to sparse channel estimation by sinking the correlation between column vectors of the dimension matrix to formulate an optimized dimension matrix. It can tip a further amended performance in sparse channel estimation.

Si Zhang[3] assess channel comeback of data communication over a multipath wireless channel. The author ruminate the channel estimation of frequency selective wireless channels which is the opposite of time selective wireless. Based on compressed channel sensing an optimization for channel estimation is proposed in this paper. The delay of the channel to optimize the algorithm of Orthogonal Matching Pursuit has been used. They have presented the LS and CS estimators and a method for modifications compromising of performance.

Tomasoni[4] proposed joint estimation of the channel length and of the impulse response for OFDM systems, developing information criteria, to find the paramount trade-off between noise rejection and channel depiction accuracy. Focusing on the 802.11 wireless LAN family, author investigate several cases, applying the joint channel length and impulse response estimation to many scenarios, ranging from the simplest pilot-aided channel estimation based on training sequences, to the most challenging data-aided channel tracking, driven either by detected or decoded symbols.

Jung-Chieh[5] investigates the pilot placement problem for sparse channel estimation in orthogonal frequency division multiplexing (OFDM) systems. Prompted by the success of the compressed sensing modus operandi in convalescing sparse signals from under sampled measurements, compressed sensing has been successfully applied for pilotaided sparse channel estimation in OFDM systems to reduce the transmitted overhead. To lessen the computational complication and at the same time maximize the accuracy of sparse channel estimation, cross-entropy optimization is introduced to determine the optimal pilot placement. Computer simulation results demonstrate that the pilot index sequences obtained using the proposed method performed better compared with those obtained using the conventional equispaced scheme and the random search method.

After reviewing existing research work in this area it seems that an efficient Pilot Design Scheme for Sparse Channel Estimation in OFDM Systems is required which have higher signal to noise ratio, low bit error rate and less energy spectrum leakage problem. CEO Approach for Sparse Channel Offset Estimation in OFDM Systems having particle impoverishment problem i.e. particles having higher weight statistically selected many times which is inappropriate for performance enhancement. Thus there arise needs of a scheme which overcome this entire problem.

\section{PROPOSED SOLUTION}

In this paper proposed solution incorporate DFT Based Superimposed Pilot Aided approach with existing Crossentropy optimization (CEO) Approach for Sparse Channel Offset Estimation in OFDM Systems in order to detain particle impoverishment problem.
For CEO estimation in OFDM in this paper a pilot based Hamming window filtering approach has been proposed which employed pilot tone insertion mechanism for Channel domain and transmit every OFDM symbol to reduce the transmitted overhead. Towards the CEO estimation in OFDM initially Pilot tones are inserted into the Channel domain and transmit each OFDM symbol for optimized CEO estimation. Consider an OFDM system with $\mathrm{N}$ subcarriers. Out of these subcarriers, $\mathrm{n}$ subcarriers are selected as pilot subcarriers which are known at the receiver. For ease of notation, the positions of the selected subset of pilot tones can be represented by

$p=$

$\left\{p_{n}\right\}_{n=0}^{N-1}$

where $p_{n} \in\{0.1\}$, n stands for the index of the subcarriers, and $\mathrm{p}^{\mathrm{n}}$ denotes whether the $\mathrm{n}^{\text {th }}$ subcarrier is selected.Proposed scheme has been used to combine frequency domain pilot spacing pattern. The pilot assembly of the channel frequency is discriminatively sensitive to channel, which assist to overwhelm the antagonistic effects of fast fading. Carrier for apiece OFDM symbol is the pilot number of $\mathrm{N}$, which is integer multiple of the number of $\mathrm{M}$, and each symbol is the pilot of the first point of the carrier.

To further reduce the OFDM channel estimation complexity, this paper adopt CEO based DFT-based interpolation algorithm, explained in Figure 4. The basic idea of whose Algorithm is zero interpolation in the frequency domain signal is equivalent to interpolation in the wavelength, thereby restoring the channel frequency comeback. DFT has a profligate algorithm FFT, so its implementation complexity can be reduced.

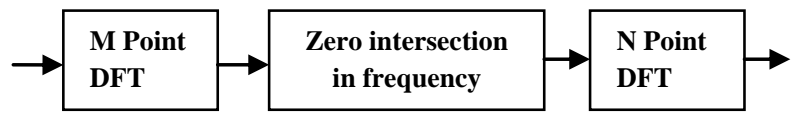

Figure 4: Channel estimation based on DFT interpolation

Through DFT (Where $\mathrm{P}_{\mathrm{L}}[\mathrm{i}]$ denotes the $\mathrm{L}^{\text {th }}$ transmit symbol at $\mathrm{i}^{\text {th }}$ sub-carrier) on or after where pilot tones are extracted. Once the estimation of CEO from pilot tones in the Channel domain $\alpha$ (i.e. Channel offset estimation) is done as show in equation 2 , then the signal is remunerated with the estimation of CEO in the time domain also. In order to precede this process CEO estimation are implemented into two different modes namely acquisition and tracking modes. Acquisition mode is responsible for estimating large range of CEO, whereas tracking mode responsible only for fine CEO estimation.

$$
\begin{aligned}
& \dot{\alpha}=\left(\frac{1}{2 \pi T_{S U B}}\right) \max (\alpha) \\
& \left\{\sum_{j=0}^{l-1} p_{l+d}[a[j], \alpha] p_{l}^{*}[a[j], \alpha] q_{l+d}^{*}[a[j]] q_{l}[a[j]\right.
\end{aligned}
$$

Where $1, a[j]$ and $\mathrm{q}[\mathrm{a}[\mathrm{j}]]$ represent number of pilot tone, location of Jth pilot tone and $\mathrm{q}$ is the pilot tone located at a[j] location in the Channel domain at $1^{\text {th }}$ symbol periods.

Then filter particles approach Gauss (PF) to estimate the carrier Channel offset (CEO) systems.PF in OFDM is stronger especially for nonlinear problems where traditional approaches like maximum likelihood estimators cannot show the best performance. PF suffers the problem of reducing the standard particles (PI) due to re-sampling the static parameter (i.e. CEO) estimation. Hamming window PF 
(HWPF) inhibits IP problem because the process of resampling is not required in the algorithm. The HWPF approximates filtration and predictive Hamming window densities on a frame PF. The HWPF updated recursively as posterior mean and covariance of the parameter of interest (i.e. CEO). The basic idea is to represent a PF density (for example filtering or higher density) samples generated and their associated weights. Then approximate particle density filtration and weight is displayed. The approximation of the density of particles filtered and weight as

$p\left(x_{k} \mid y_{k}\right)=\sum_{i=1}^{M} w_{k}^{i} \alpha\left(x_{k}-\right.$

$x k i)$......

Where i represent particle and $\mathrm{k}$ represent its time index whereas $\mathrm{M}$ denote total particle number. Then on the basis of that particles weight proposed approach estimate as show in algorithm.

\section{Assumption \\ $\mathrm{S}_{\mathrm{OFDM}}=$ Input signal OFDM \\ $\mathrm{P}_{\mathrm{T}}=$ Pilot tone \\ $\mathrm{F}_{\mathrm{d}}=$ frequency domain of $\mathrm{S}_{\mathrm{OFDM}}$}

\section{Algorithm}

Step 1:- OFDM initially Pilot tones are inserted into the frequency domain

$$
S_{\text {OFDM }}^{P_{T}}=\operatorname{DFT}\left(S_{\text {OFDM }}\right)+
$$$$
P_{T}[i]_{i=0}^{x-1}
$$

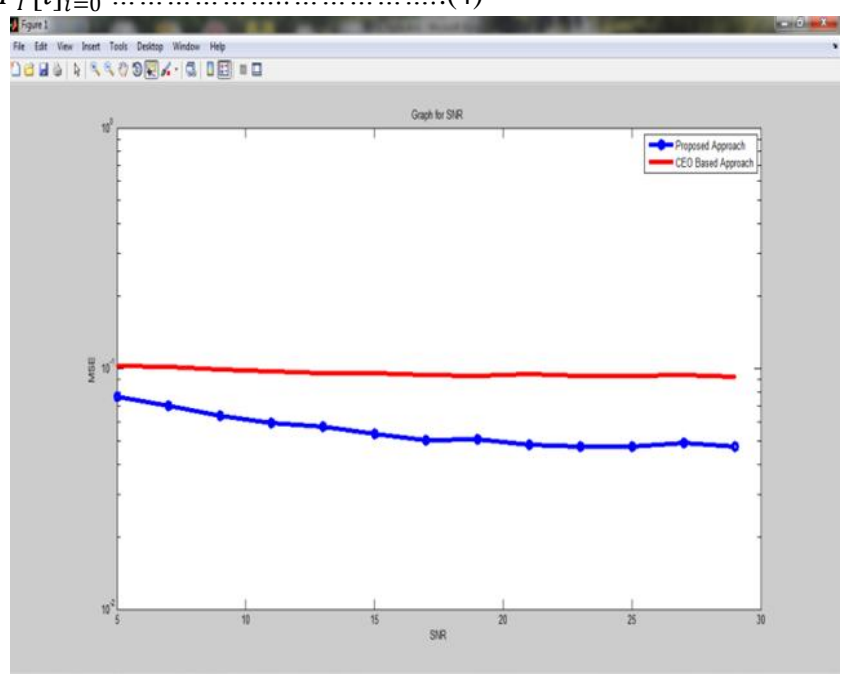

Figure 5(a):- $(\mathbf{N}, \mathbf{L}, \mathrm{F}, \mathrm{K})=\mathbf{2 5 6 , 1 0 0 , 1 1 . 4 , 1 6}$
Step 2:- Transmit each frequency domain $\alpha$ (OFDM symbol) for SFO tracking

$$
\begin{gathered}
\dot{\alpha}=\left(\frac{1}{2 \pi T_{S U B}}\right) \max (\alpha) \\
\left\{\sum_{j=0}^{l-1} p_{l+d}[a[j], \alpha] p_{l}^{*}[a[j], \alpha] q_{l+d}^{*}[a[j]] q_{l}[a[j]\right.
\end{gathered}
$$

Step 3:- Apply Hamming window particle Filter approach (PF) to estimate the sparse Channel offset (SCO) systems and approximation of the density of particles filtered and weight as

$$
p\left(x_{k} \mid y_{k}\right)=\sum_{i=1}^{M} w_{k}^{i} \alpha\left(x_{k}-\right.
$$$$
\left.x_{k}^{i}\right) \text {. . . . }
$$

Step 4:-Then on the basis of that particles weight proposed approach estimate $\mathrm{SCO}$ for frequency offset.

\section{SIMULATION AND PERFORMANCE EVALUATION}

This section provides simulation results for the evaluation of the proposed algorithm for Sparse Channel Estimation from an OFDM system. Simulation scenario use ITU vehicular channel model [8] to simulate a sparse multipath channel. The graph of SNR and MSE are generated by taking four parameters. They are number of subcarriers $(\mathrm{N})$, length of channel (L), sampling frequency (F) and modulation order $(\mathrm{K})$.

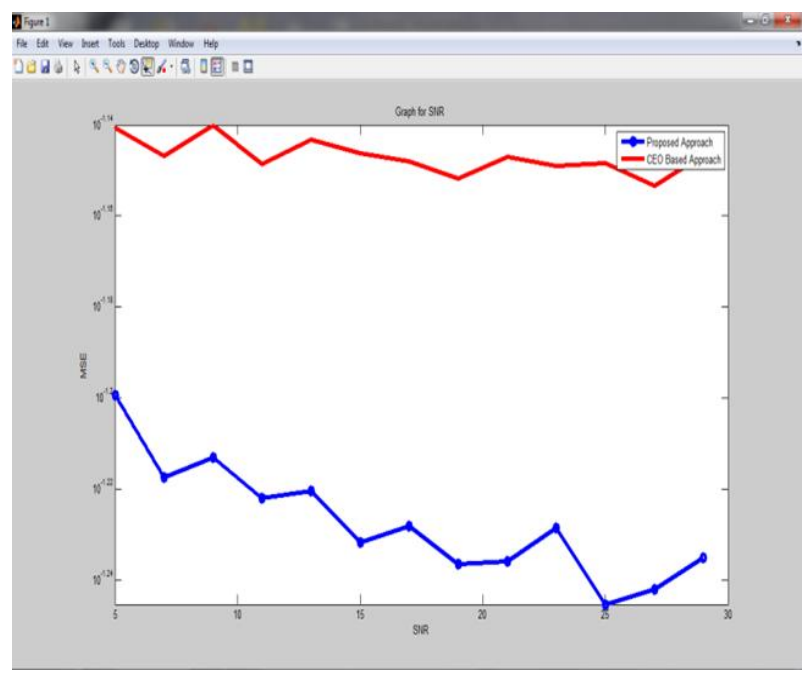

Figure 5(b):- $(\mathrm{N}, \mathrm{L}, \mathrm{F}, \mathrm{K})=\mathbf{5 1 2 , 1 0 0 , 1 1 . 4 , 3 2}$

Figure 5: SNR

Figure 5 gives the comparison of SNR. The Signal-to-noise ratio (abbreviated SNR) is a measure used to compare the level of a desired signal to the level of background noise. It is precise as the ratio of signal power to the noise power, often expressed in decibels. From figure 5 it can be concluded that the proposed technique gives better result than the previous CEO based technique. Figure 6 shows the graph of MSE performances. It represents that the MSE obtained from the hybrid approach that is the use of DFT based superimposed pilot aided approach with existing CEO approach is comparatively less than MSE obtained by only CEO technique. Figure 6 plot channel mean square error with EsNodB. MSE performance of the proposed design for different values of $\mathrm{N}, \mathrm{K}, \mathrm{L}$ and $\mathrm{F}$ are shown in Fig 6 (a) and Figure 6 (b).

$\mathrm{Es} / \mathrm{NodB}=\mathrm{Eb} / \mathrm{NodB}+10 * \log 10(\mathrm{nSC} / \mathrm{nFFT})+10 * \log 10$ $(\mathrm{Td} / \mathrm{Td}+\mathrm{Tc})+10 * \log 10(\mathrm{k})$

The sparse channel estimates are calculated from pilot observations by using a practical compressed sensing algorithm. Mean squared error of the different trends in terms of mean square error (MSE) of the estimator measures and that is, the estimated difference between the 
estimate and the mean square of the "errors" difference. Where nSC is No. of subcarriers, Tc is cyclic prefix duration, Td is Data symbol duration, Ts is Total Symbol duration and $\mathrm{k}$ equals $\log 2(\mathrm{M})$. MSE is a risk function, corresponding to the expected loss of error or loss of value of second class squared. The difference is caused by chance or because the estimator does not take into account information that can produce more accurate estimate.In Figure 7 and Figure 8 to obtain the graph of SNR and MSE, the parameters N, L, K are kept constant with 64,1 , and 16 respectively with different fading conditions by varying the Doppler shift frequency $\mathrm{F}$ as
150,200 and 250. Then MSE is analyzed with respect to SNR and EsNodB and are plotted in figure 7 and 8 respectively.

It is observed that:

(a) Proposed OFDM method with pilot aided channel estimate performs better than CEO with equispaced pilot.

(b) If Doppler shift frequency is increased then MSE with proposed method decreases.

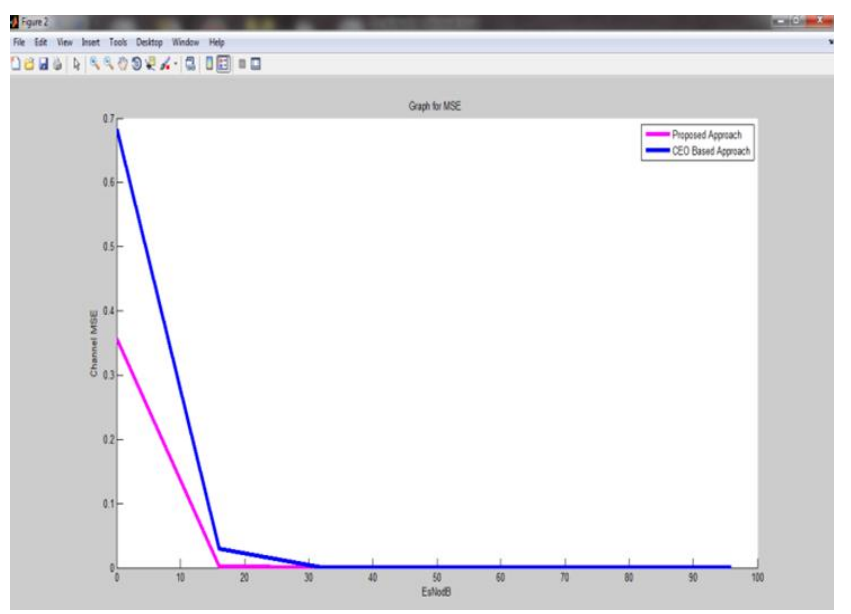

Figure 6(a):- $(\mathrm{N}, \mathrm{L}, \mathrm{F}, \mathrm{K})=256,100,11.4,16$

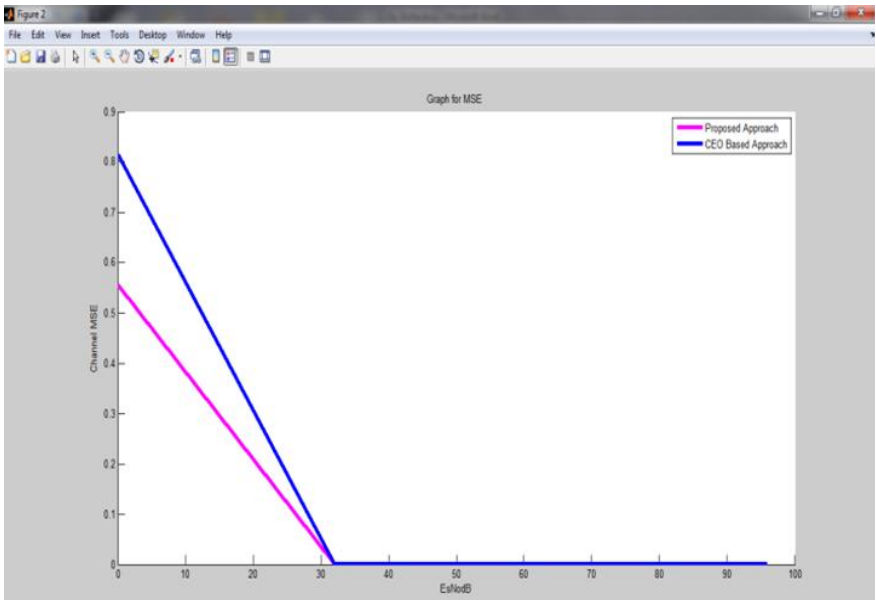

Figure 6(b):- $(\mathrm{N}, \mathrm{L}, \mathrm{F}, \mathrm{K})=\mathbf{5 1 2 , 1 0 0 , 1 1 . 4 , 3 2}$

Figure 6: MSE

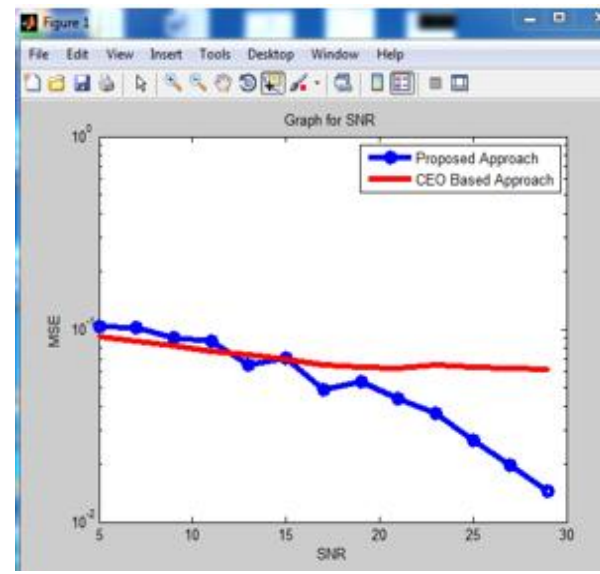

Figure 7(a): For $\mathrm{F}=150$

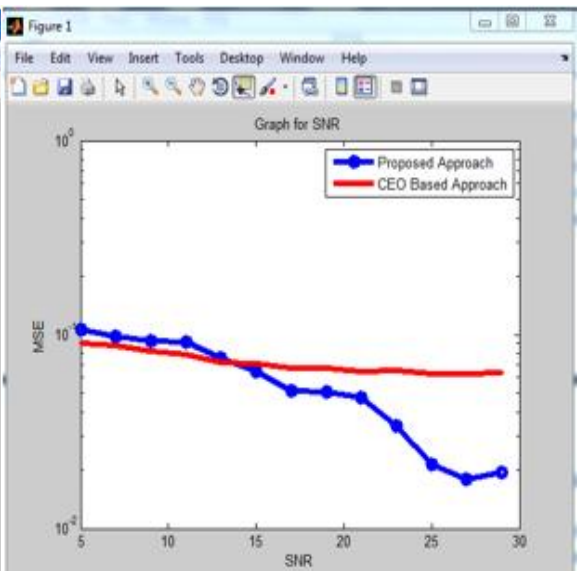

Figure 7(b): For $\mathbf{F}=\mathbf{2 0 0}$

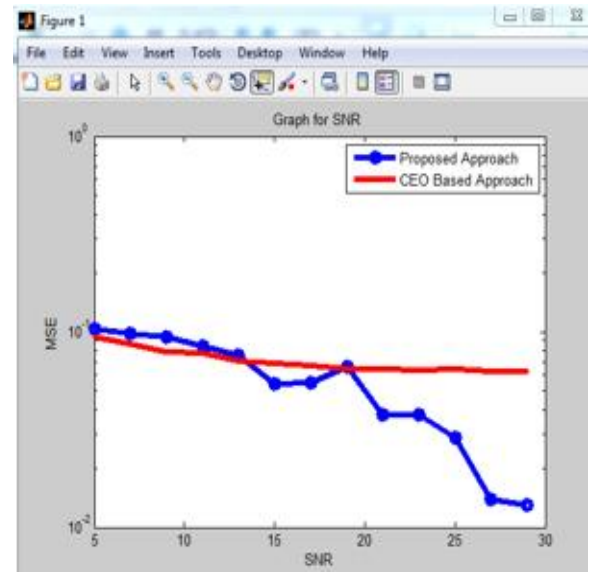

Figure 7(c): For $\mathbf{F}=250$

Figure 7: Analysis of SNR with different Doppler shifts for multipath fading 


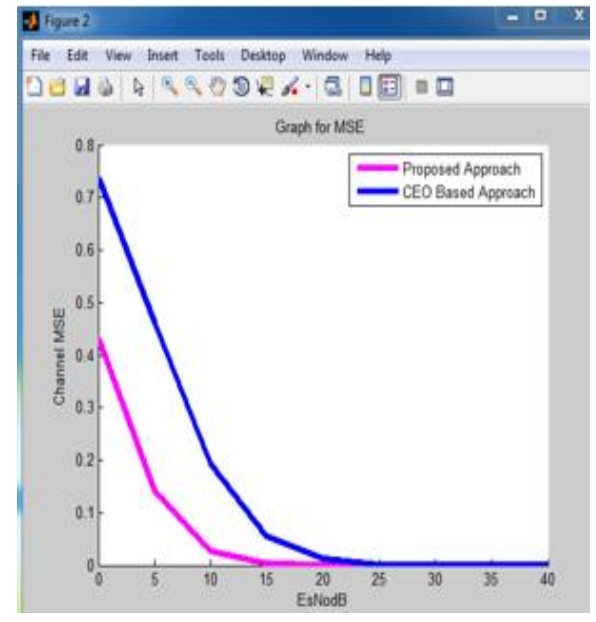

Figure 8(a):- For $\mathrm{F}=150$

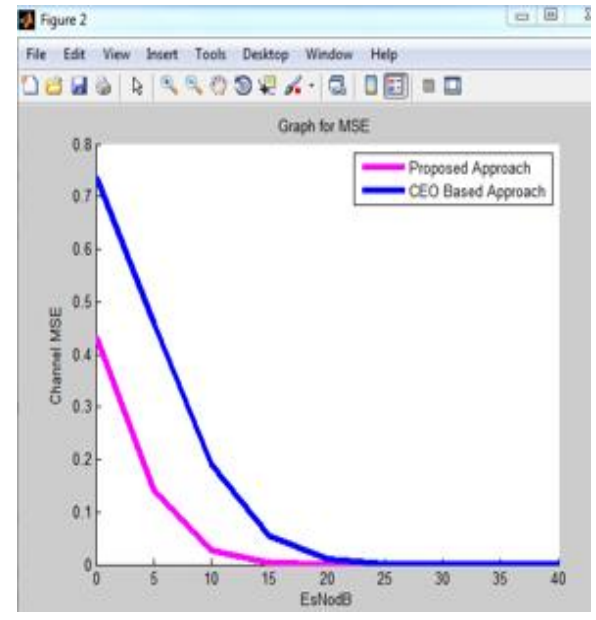

Figure 8(b):- For $F=200$

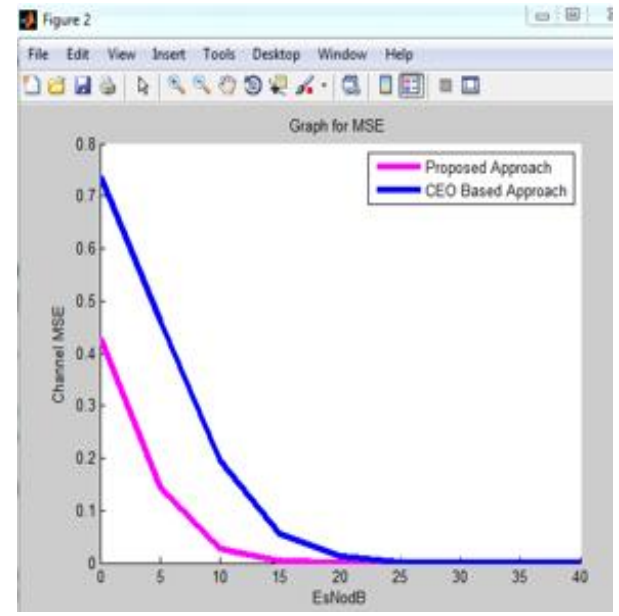

Figure 8(c):- For $\mathrm{F}=250$

Figure 8: Analysis of MSE with different Doppler shifts with multipath fading

\section{CONCLUSION}

OFDM is an emerging field in the world of wireless communication. In this way there are lots of challenges in front of us. This paper is a brief description on the OFDM and their issues. There are lots of problems that are faced in the channel offset estimation. In this paper, a pilot based hybrid approach for SCO estimation has been proposed. Pilot tone insertion mechanism for Channel domain and transmit every OFDM symbol for SCO tracking. Then Hamming window filter particle approach to estimate the sparse channel offset (SCO) systems. The proposed hybrid approach shows the outperforming results for the highly non-linear problem over all range of levels for the static parameter estimation by taking advantage of its robustness against PI problem.

\section{FUTURE SCOPE}

In future frequency offset estimator may be utilizing the periodicity embedded in a training symbol and generalize the well-known correlation-based methods such that the estimation range of all the correlations embedded in a training symbol can be extended to the maximum.

\section{REFERENCES}

[1]. Chenhao Qi and Lenan Wu, "Optimized Pilot Placement for Sparse Channel Estimation in OFDM Systems", IEEE 2011, Volume: 18 ,Issue: 12 pp 749 - 752.

[2]. Xiaochao Xiao Baoyu Zheng ; Chenhao Wang, "Compressed channel estimation based on optimized measurement matrix", IEEE 2011, pp 1-5.

[3]. Si Zhang ; Jian Kang; Yichuan Song ; Nannan Wang "An Optimization for Channel Estimation Based on Compressed Channel Sensing", IEEE 2012, pp 597-602.

[4]. Tomasoni, A.;Bellini, S. ; Ferrari, M. ; Gatti, D. , "Efficient OFDM channel estimation via an information criterion", IEEE 2012, pp 3936-3941.

[5]. Jung-Chieh Chen, Chao-Kai Wen and Pangan Ting, "An Efficient Pilot Design Scheme for Sparse Channel
Estimation in OFDM Systems", IEEE 2013, VOL. 17, NO. 7, pp 1352-1355.

[6]. R. V. Nee and R. Prasad, "OFDM for Wireless Multimedia Communications", Artech House, pp.229 231, 2000.

[7]. A.P.Palamides andA.M.Maras,"A Bayesian state-space approach to combat inter-carrier interference in OFDM systems," IEEE Signal Process. Lett., vol. 14, no. 10, pp. 677-679, Oct. 2007.

[8]. J. Lim and D. Hong, "Inter-carrier interference estimation in OFDM systems with unknown noise distributions," IEEE Signal Process. Lett.vol. 16, no. 6, pp. 493-496, Jun. 2009.

[9]. P. H. Moose, "A technique for orthogonal frequency division multiplexing frequency offset correction", IEEE Transactions on Communications, Vol. 42, No. 10, October 1994.

[10]. J.A.C. Bingham, "Multicarrier modulation for data transmission: an idea whose time has come," IEEE Commun. Mag., vol. 28, no. 5, pp. 5-14,May 1990.

[11]. Y. Yingwei and G. B. Giannakis, "Blind carrier frequency offset estimation in SISO, MIMO, and multiuser OFDM systems," IEEE Trans. Commun., vol. 53, pp. 173-183, Jan. 2005.

[12]. Z. Xiang and A. Ghrayeb, "A blind carrier frequency offset estimation scheme for OFDM systems with constant modulus signaling," IEEE Trans. Commun., vol. 56, pp. 1032-1037, 2008.

[13]. Zhongshan Zhang, Keping Long, Ming Zhao, and Yuanan Liu, "Joint Frame Synchronization and Frequency Offset Estimationin OFDM Systems” IEEE 2005,pp389-394

[14]. Praween Kumar Nishad and P. Singh, "Carrier Frequency Offset Estimation in OFDM Systems", IEEE 2013pp 885889. 